\title{
REVISION OF THE LICHEN GENUS Buellia De Not. (FAMILY PHYSCIACEAE) FROM INDIA
}

\author{
SUBHASH CHANDRA ${ }^{1}$ \\ P.G. Department of Botany, Sri Murli Manohar Town P.G. College, Ballia, Uttar Pradesh, India
}

\begin{abstract}
The paper reports seventeen species of the lichen genus Buellia De Not., from India along with a key for identification of different Indian species. Out of these, nine species, Buellia aggredians (Stirt.) Zahlbr., B. betulinoides Schubert \& Klement, $B$. inornata (Stirt.) Zahlbr., B. lauricassiae (Fèe) Müll. Arg., Müller, B. manipurensis Singh and Singh, B. meghalayensis S. Singh and D. D. Awasthi, B. morehensis Singh and Singh, B. montana Magn., and B. palniensis S. Singh and D. D. Awasthi are reported as endemic species for Indian lichen flora.
\end{abstract}

KEYWORDS: Buellia, Lichen, Key, Endemic species and Distribution

During the course of revisionary studies on the lichen family Physciaceae under "Flora of India" project, several lichen specimens collected earlier from Eastern, North-Eastern, Western and Southern regions of India have been investigated. The lichen genus Buellia De Not., is characterized by thallus crustose, continuous, rimose areolate, granulose or verrucose, rarely sorediate or isidiate, hypothallus mostly as a black or brownish line along the margine or in between the thalli, rarely absent. Thallus thin or moderately thick, cortex not well differentiated phycobiontic cells spherical to ellipsoidal, green, \pm uniformly distributed. Apothecia immersed, adnate or sessile, round or rarely angular or irregular, margin indistinct or prominent, disc black, plane slightly or strongly convex, epruinose or rarely pruinose, exciple thin or moderately thick, dark brown to black or dark brown on outer side and pale brown or hyaline on inner side or completely pallid, epithecium brown to dark brown or olive brown, hymenium hyaline, with or without oil globules, hypothecium hyaline to pale brown, internal stipe distinct or indistinct, pallid, reddish brown or dark brown, asci clavate, 8 -spored or occasionally 12-16 spored, ascospores brown, 2-celled, ellipsoid, rarely ovoid, straight or curved, unconstructed or constricted at septum and not surrounded by a gelatinous halo, spore wall uniformly or unevenly thickened, surface smooth or ornamented, paraphyses simple to branched, apically swollen and brown (Awasthi, 1988; Singh and Singh, 1982; Singh and Awasthi, 1981).

\section{MATERIALS AND METHODS}

This study is based on consultation of herbarium materials that are preserved in different lichen herbarium at Agharkar Mycological Herbarium (AMH), Agharkar
Research Institute, Pune; National Botanical Research Institute, Lucknow (LWG); Botanical Survey of India, Eastern Circle, Shillong (ASSAM). Central National Herbarium (CNH), Botanical Survey of India, Kolkata (CAL). The materials were brought on loan from the above lichen herbariums to Botanical Survey of India, Central Circle, Allahabad (BSA).

The specimens were investigated morphologically, anatomically and chemically. The colour tests were performed with the usual reagents i.e. K (5\% Potassium hydroxide), C (aqueous solution of hypochlorite) and PD (Paraphenylene diamine). Lichen substances were investigated with thin layer chromatography (TLC) in solution A (180 toluene: 60 dioxane: 8 acetic acid) using the technique of Culberson (1972) and Walker and James (1980).

\section{Key to the Indian species of genus Buellia}

1a.Thallus saxicolous.....................................

1b. Thallus corticolous..................................9

2a. Spore wall ornamented on surface........................ 3

2b. Spore wall smooth on surface........................ 8

3a. Thallus $\mathrm{K}^{+}$yellow or yellow red........................4

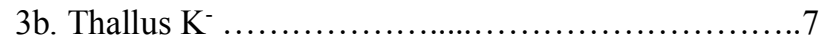

4a. Thallus surface smooth..............................5

4b. Thallus surface rough................................. 6

5a. Thallus glaucous white to grey, areolate, deeply fissured, hypothallus absent, $\mathrm{K}^{+}$red, $\mathrm{PD}^{+}$ yellow............................................ ceylensis 


\section{CHANDRA: REVISION OF THE LICHEN GENUS Buellia De Not. (FAMILY PHYSCIACEAE) FROM INDIA}

5b. Thallus yellowish, grey to ashy grey, deeply fissured, hypothallus black line along the margin, $\mathrm{K}^{+}$yellow, $\mathrm{PD}^{+}$deep yellow. B. meghalayensis

6a. Thallus greyish brown, cracked to areolate, hypothallus dark brown, thick, $2-4$ celled ascospore, $\mathrm{K}^{+}$yellow to reddish brown, $\quad \mathrm{PD}^{+}$deep yellow................................................ manipurensis

6b. Thallus grey to grey brown, verruculose, hypothallus indistinct but blackish at its junction, $\mathrm{K}^{+}$red, $\mathrm{PD}^{+}$ yellow. B. morehensis

7a. Thallus dull brownish, grey, subsquamulose, rough, hypothallus absent, $\mathrm{K}^{-}, \mathrm{PD}^{-}$ B. palniensis

7b. Thallus brownish grey, rough, hypothallus indistinct, mischoblastiomorphic type of ascospore, $\mathrm{K}^{+}$yellowish, $\mathrm{PD}$ B. posthabita

8a. Spore wall ornamented on surface. 10

8 b. Spore wall smooth on surface. 15

9a. Thallus $\mathrm{K}^{+}$yellow or yellow to red .11

9b. Thallus $\mathrm{K}^{-}$ 13

10a. Thallus rough, greyish white to grey granulose to verruculose, mischoblastiomorphic type of ascospore, $\mathrm{K}^{+}$ yellow turning red, $\mathrm{PD}^{+}$yellow. B. inornata

10b. Thallus smooth 12

11a. Thallus whitish grey to glaucous white, subverruculose to granulose, deeply fissured, $\mathrm{K}^{+}$yellow, $\mathrm{PD}^{+}$ yellow.... B. punctata

11b. Thallus whitish grey to grey, weakly fissured, $\mathrm{K}^{+}$ yellow, PD B. montana

12a. Thallus smooth, sorediate, soredia dull whitish, farinose. B. betulinoides

12b. Thallus rough, soredia absent .14

13a. Thallus glaucous white to greyish white, subsquamulose, weakly fissured, $\mathrm{K}^{-}$................... curtisii

13b. Thallus greyish brown to grey, subverruculose, weakly fissured, placodiomorphic type of ascospore, $\mathrm{K}^{-}$ .B. placodiomorpha

14a. Thallus smooth, greyish white to grey, weakly fissured, $\mathrm{K}^{+}$yellowish, $\mathrm{PD}$ B. aggredians 14b. Thallus rough 16

15a. Thallus grey to brownish grey, deeply fissured, $\mathrm{K}^{+}$ yellow turning red, $\mathrm{PD}^{+}$yellow- orange....... B. disciformis

$15 \mathrm{~b}$. Thallus whitish grey to grey, verruculose to granulose, weakly fissured, $\mathrm{K}^{+}$yellow turning red, $\mathrm{PD}$ B. glaucotheca

16a. Thallus greyish white, subverruculose, weakly fissured, 6-celled, 3-transversly and 2- longitudinal septate, submuriform of ascospore, $\mathrm{K}^{+}$yellow, $\mathrm{PD}^{-}$ B. alboatra

16b. Thallus glaucous grey to grey, verruculose, weakly fissured, 4-celled, 3-transversely septate ascospore, $\mathrm{K}^{+}$ yellow turning red, $\mathrm{PD}^{+}$yellow-orange.....B. lauricassiae

\section{RESULTS AND DISCUSSION}

\section{Taxonomic Descriptions}

Buellia aggredians (Stirt.) Zahlbr., Cat. Lich. Univ., 7: 333, 1931; Lecidea aggredians Stirt., Proc. Phil. Soc., Glasgow, 11: 313, 1879.

Type: India; Neilgherries (Nilgiri), on bark of tree, G. Watt, s. n. (BM), (not seen).

Thallus corticolous, crustaceous, $2-3 \mathrm{~cm}$ across, whitish grey to grey, verruculose, surface smooth, weakly fissured, epruinose, hypothallus black delimiting line along the margin, isidia and soredia absent. Apothecia sessile, immersed, 0.2-0.5 $\mathrm{mm}$ diameter, disc black, plane to convex, covered with dense pruina, oil globules present, epithecium brown $24 \mu \mathrm{m}$ thick, hymenium hyaline, 96-108 $\mu \mathrm{m}$ high, insert with oil globules, hypothecium dark brown 108-120 $\mu \mathrm{m}$ thick, exciple dark brown $60-84 \mu \mathrm{m}$ thick, asci 8 -spored, clavate, $60-72 \times 12-18 \mu \mathrm{m}$, ascospores brown, 2celled, ellipsoid, $15-28 \times 6-9 \mu \mathrm{m}$ in size, wall uniformly thickened, surface ornamented, paraphyses hyaline, simple, globose at tips.

Chemistry: Thallus $\mathrm{K}^{+}$yellowish, $\mathrm{C}^{+}$reddish to red, $\mathrm{KC}^{+}$ red, $P D$

Remark: Buellia aggredians resembles B. glaucotheca (Fèe) Malme and B. epimarta (Nyl.) Malm, which are distinguished by different thallus reactions and absence of oil globules in hymenium.

Distribution: India (Tamil Nadu and West Bengal). 


\section{CHANDRA: REVISION OF THE LICHEN GENUS Buellia De Not. (FAMILY PHYSCIACEAE) FROM INDIA}

Specimens examined: West Bengal, 24-Parganas, Saptagram, Birati, 16.9.1966, K. N. Roychowdhury 395 (CAL); Woodlands, alt. 4500 ft., 16.9.1967, C. G. Dharne \& K. N. Roychowdhury 1341 (CAL); Galla road side, 12.2.1970, K. N. Roychowdhury 1690 (CAL).

Buellia alboatra (Hoffm.) Th. Fr., Fries 91: 1861; Lichen alboatra Hoffm., 30: 1784; Lichen corticola Acharius, nom. Superfl., 131: 1795; Abacina alboatra (Hoffm.) Norman, 236: 1853; Diplotomma alboatrum (Hoffm.) Flotow, 380, 382: 1850; Rhizocarpon alboatrum (Hoffm.) Anzi, 92: 1860.

Type: Germany, (Arnold, Lich. Mon 55: UPS), (not seen).

Thallus corticolous, crustaceous, 2-3 cm across, whitish grey, subverruculose to granulose, areolate, surface rough, weakly fissured, epruinose, hypothallus indistinct, isidia and soredia absent. Apothecia sessile, immersed, 0.2$0.5 \mathrm{~mm}$ diameter, disc black, plane to convex, slightly pruinose, oil globules absent, epithecium pale brown 24-36 $\mu \mathrm{m}$ thick; hymenium hyaline $84-108 \mu \mathrm{m}$ high, hypothecium dark brown 84-96 $\mu \mathrm{m}$ thick; exciple dark brown 60-72 $\mu \mathrm{m}$ thick; asci 8-spored, clavate, 60-72 x 12-18 $\mu \mathrm{m}$, ascospores brown, 6-celled, 3-transversely and 2-longitudinal septate, submuriform, 15-24 x 6-12 $\mu \mathrm{m}$ in size, wall uniformly thickened, surface smooth, paraphyses hyaline, simple, globose at tips.

Chemistry: Thallus $\mathrm{K}^{+}$yellowish, $\mathrm{C}^{-}, \mathrm{KC}^{-}, \mathrm{PD}^{-}$

Remark: B. alboatra is distinguished by 6-celled, 3transversely and 2-longitudinal septate and submuriform of ascospores and $\mathrm{K}^{+}$yellow thalli.

Distribution: India (Manipur); Europe, North America, Africa, Australia and New Zealand.

Specimens examined: Manipur, Litan, Sakkibung, alt. ca. 3500-4000 ft., 14.11.1976, K. P. Singh 54912 (CAL).

\section{Buellia betulinoides Schubert \& Klement, Nova Hedwigia, 11} (1-4): 66, 1966.

Type: India; Jammu \& Kashmir, Batote, alt. 1600 m., on Cedrus (Hb. Klement), (not seen).

Thallus corticolous, crustaceous, 2-3 cm across, greyish brown, subverruculose to granulose, surface smooth, weakly fissured, epruinose, sorediate, sorelia dull whitish, farinose, hypothallus absent. Apothecia sessile, immersed, 0.2-1.0 $\mathrm{mm}$ diameter, disc black, plane to convex, epruinose, oil globules absent, epithecium pale brown $24 \mu \mathrm{m}$ thick, hymenium hyaline 84-96 $\mu \mathrm{m}$ high, hypothecium dark brown 84-96 $\mu \mathrm{m}$ thick, exciple brown on outer and pallid on inner side, 48-60 $\mu \mathrm{m}$ thick, asci 8spored, unitunicate, ascospores brown, 2-celled, ellipsoid, 15-24 × 6-9 $\mu \mathrm{m}$ in size, wall uniformly thickened, surface ornamented, paraphyses hyaline, simple, globose at tips.

Chemistry: Thallus $\mathrm{K}^{-}, \mathrm{C}^{-}, \mathrm{KC}^{-}, \mathrm{PD}^{-}$

Remark: B. betulinoides is distinguished by presence of dull whitish farinose, soredia on the thalli.

Distribution: India (Manipur and Tamil Nadu).

Specimens examined: Manipur, Saraikong, Litan, alt. ca. 3600 ft., 9.11.1976, K. P. Singh 54782 (CAL); Karang, Kabru lakha area, alt. ca. 4000-5000 ft., 1.11. 1976, K. P. Singh 55044 (CAL).

Buellia ceylanensis Zahlbr., Zahlbr. Apud Rechinger, Deukschrift. Math. Naturv. Class Kais. Acad. Wiss Wien, 18: 31, 1911; Awasthi \& Singh, Geophytology, 1(2): 101, 1972 .

Type: Ceylon (Sri Lanka), near Kandy, on quartz, Rachinger 2768, (W), (not seen).

Thallus corticolous, crustaceous, $3-4 \mathrm{~cm}$ cross, grey to greyish brown, areolate, surface smooth, deeply fissured, bright, epruinose, hypothallus indistinct, isidia and soredia absent. Apothecia sessile, immersed, 0.2-1.0 mm diameter, disc black, plane to convex, slightly pruinose, oil globules absent, epithecium brown $24 \mu \mathrm{m}$ thick, hymenium hyaline 72-84 $\mu \mathrm{m}$ thick, hypothecium pale brown $60-72 \mu \mathrm{m}$ thick, exciple dark brown on outer side and pallid on inner side 72-96 $\mu \mathrm{m}$ thick, asci 8-spored, unitunicate, ascospores brown, 2-celled, ellipsoid, 18-24 x 6-12 $\mu \mathrm{m}$ in size, wall uniformly thickened, surface ornamented, paraphyses hyaline, septate, branched, globose at tips.

Chemistry: Thallus $\mathrm{K}^{+}$red, $\mathrm{C}^{-}, \mathrm{KC}^{-}, \mathrm{PD}^{+}$deep yellow.

Remark: B. ceylanensis resembles B. meghalayensis but the latter is distinguished by $\mathrm{K}^{+}$red thalli and larger spore size.

Distribution: India (Manipur and Tamil Nadu); Ceylon

Specimens examined: Manipur, Mao, alt. 6200ft., 5.6.1979, K. P. Singh 550368 (CAL).

Buellia curtisii (Tuck.) Imsh. In Brodo, Lichens of Long Island, New York; 253, 1968. Gyrostomum curtisii Tuck. Am. J. Arts Sci. 2(25): 430, 1858.

Type: North Carolina, Trunks, Dr. Curtis (not seen). 


\section{CHANDRA: REVISION OF THE LICHEN GENUS Buellia De Not. (FAMILY PHYSCIACEAE) FROM INDIA}

Thallus corticolous, crustaceous, 3-4 cm across, glaucous white to greyish white, verruculose, subsquamulose, surface rough, weakly fissured, epruinose, hypothallus indistinct, isidia and soredia absent. Apothecia sessile, immersed, 0.2-0.5 mm diameter, disc black, plane to convex, epruinose, oil globules absent, epithecium brown $24 \mu \mathrm{m}$ thick, hymenium hyaline 84-96 $\mu \mathrm{m}$ high, hypothecium pale brown 96-108 $\mu$ m thick, exciple brown on outer and pallid on inner side 36-45 $\mu \mathrm{m}$ thick, asci 8spored, unitunicate, ascospores brown, 2-celled, ellipsoid, 15-28 $\times$ 6-9 $\mu \mathrm{m}$ in size, wall uniformly thickened, surface ornamented, paraphyses hyaline, septate, branched, globose at tips.

Chemistry: Thallus $\mathrm{K}^{+}$yellow to red brown, $\mathrm{C}^{-}, \mathrm{KC}^{-}, \mathrm{PD}^{+}$ deep yellow to reddish.

Remark: B. curtisii resembles $B$. stilligiana which is distinguished by dark brown exciple, giving red crystals in $\mathrm{K}$ solution and presence of zeorin in the thalli.

Distribution: India (Manipur); North America.

Specimens examined: Manipur, Karang, Kabru-Lakha area, alt. 3500 ft., 14.6.1979, K. P. Singh 55083 (CAL); Ukhrul, Carnation Avenue, alt. ca. 5500 ft., 4.11.1976, K. P. Singh 55149 (CAL).

Buellia disciformis (Fr.) Mudd., Man. Brit. Lich. 1861. Sheard, Lichenologist, 2: 239, 1964. Lecidea parasema var. disciformis Fr., Nov. Sched. Critic, 9, 1826.

Type: Stirp. Crypt. Vog. Rhem., Maugeot et Nestler 745 (UPS), (not seen).

Thallus corticolous, crustaceous, $4-5 \mathrm{~cm}$ across, grey to brownish grey, subverruculose, surface smooth, deeply fissured, epruinose, hypothallus dark brown to black delimiting line around the thallus, isidia and soredia absent. Apothecia sessile, immersed, 0.2-0.5 mm diameter, disc black, plane to convex, epruinose, oil globules present, epithecium brown $24 \mu \mathrm{m}$ thick, hymenium hyaline $72-84$ $\mu \mathrm{m}$ high, inserted with oil globules, hypothecium dark brown 96-120 $\mu \mathrm{m}$ thick, exciple dark brown 36-72 $\mu \mathrm{m}$ thick, asci 8-spored, unitunicate, ascospores brown, 2celled, ellipsoid, 15-28 x 6-9 $\mu \mathrm{m}$ in size, wall uniformly thickened, surface smooth, paraphyses hyaline, simple, globose at tips.

Chemistry: Thallus $\mathrm{K}^{+}$yellow to red, $\mathrm{C}^{-}, \mathrm{KC}^{-}, \mathrm{PD}^{+}$yelloworange.
Remark: B. disciformis is distinguished by different thallus reactions $\mathrm{K}^{+}$yellow turning red, $\mathrm{PD}^{+}$yellow-orange and presence of oil globules in hymenium.

Distribution: India (Manipur and West Bengal).

Specimens examined: Manipur, Ukhrul, on way to Tori, alt. 3500-4000 ft., 7.11.1976, K. P. Singh 54717 (CAL); West Bengal, 24-Paragnas, Chowngda (Halera), 27.11.1970, K. N. Roychowdhury 1409, 1429 (CAL).

Buellia glaucotheca (Fèe.) Malme, Ark. Bot. 21 A (14): 20, 1927. Imsh., Farlowia, 4: 491, 1955. Lecidea glaucotheca Fèe., Suppl. Essai. Crypt. Ecorc. Office, 109, 1837.

Type: Habitat in corticibus Cinchonae Condamineae, Humbold \& Bonpland, (not seen).

Thallus corticolous, crustaceous, 1-2 cm across, whitish to dusty grey, verruculose to granulose, surface bright, rough, weakly fissured, epruinose, hypothallus black delimiting line around the thallus, isidia and soredia absent. Apothecia sessile, immersed, 0.2-1.0 mm diameter, disc black, plane to convex, covered with white pruina, margin black, oil globules absent, epithecium brown $24 \mu \mathrm{m}$ thick, hymenium hyaline 84-96 $\mu \mathrm{m}$ high, hypothecium dark brown, $\mathrm{K}^{+}$red, 108-120 $\mu \mathrm{m}$ thick, exciple brown to dark brown 48-72 $\mu \mathrm{m}$ thick, asci 8-spored, unitunicate, ascospores brown, 2-celled, ellipsoid, 18-27 x 6-9 $\mu \mathrm{m}$ in size, wall uniformly thickened, surface smooth, paraphyses hyaline, simple, globose at tips.

Chemistry: Thallus $\mathrm{K}^{+}$yellow turning red, $\mathrm{C}^{-}, \mathrm{KC}^{-}, \mathrm{PD}^{+}$ deep yellow.

Remark: B. glaucotheca resembles B. aggredians, which is distinguished by $\mathrm{K}^{+}$red thallus and hymenium inspersed with oil globules.

Distribution: India (Manipur and Tamil Nadu); Cuba, Jamaica, South America and West Indies.

Specimens examined: Manipur, Mapith hill, range peak, alt. ca. 4500-5500 ft., 19.11.1976, K. P. Singh 54948 (CAL).

Buellia inornata (Stirt.) Zahlbr., Cat. Lich. Univ., 7: 369, 1931. Lecidea inornata Stirt., Proc. Phil. Soc. Glasgow, 3: 185, 1881.

Type: India; Assam, A. Watt s. n. ex Hb. Stirton, (BM), (not seen).

Thallus corticolous, crustaceous, 3-4 cm across, whitish grey to grey, verruculose to granulose, surface 


\section{CHANDRA: REVISION OF THE LICHEN GENUS Buellia De Not. (FAMILY PHYSCIACEAE) FROM INDIA}

rough, weakly fissured, epruinose, hypothallus brownish black delimiting line along the margin, isidia and soredia absent. Apothecia sessile, immersed, 0.2-1.0 mm diameter, disc black, plane to convex, epruinose, oil globules absent, epithecium brown $24 \mu \mathrm{m}$ thick, hymenium hyaline 108-120 $\mu \mathrm{m}$ high, hypothecium dark brown to black, $108-120 \mu \mathrm{m}$ thick, exciple brown to dark brown 60-84 $\mu$ m thick, asci 8spored, unitunicate, ascospores brown, 2-celled, mischoblastiomorphic, , 15-24 x 6-9 $\mu \mathrm{m}$ in size, wall uniformly thickened, surface ornamented, paraphyses hyaline, simple, globose at tips.

Chemistry: Thallus $\mathrm{K}^{+}$yellow turning red, $\mathrm{C}^{-}, \mathrm{KC}^{-}, \mathrm{PD}^{+}$ deep yellow.

Remark: $B$. inornata is an endemic species, widely distributed in India. In external morphology and chemistry, this taxon closely resembles $B$. lauricassiae (Fèe) Müll. Arg., but the latter possesses 4-celled ascospores.

Distribution: India (Andhra Pradesh, Assam, Bihar, Madhya Pradesh, Manipur, Tamil Nadu, Uttarakhand and West Bengal).

Specimens examined: Manipur, Tegnopal, alt. $5000 \mathrm{ft}$., 21.6.1979, K. P. Singh 550914 (CAL).

Buellia lauricassiae (Fèe) Müll. Arg., Müller, 85: 1887. Lecidea lauricassiae Fèe, 101: 1837. Mannia lauricassiae (Fèe) Trevisan, 77: 1857. Diplotomma lauricassiae (Fèe) Szatala, 123: 1932. Cratiria lauricassiae (Fèe) Marbach, 160: 2000.

Type: India; West Bengal, Kolkata, Seebpore, Kurz (UPS), (not seen).

Thallus corticolous, crustaceous, $3-4.5 \mathrm{~cm}$ across, glaucous grey to grey, verruculose to granulose, surface rough, weakly fissured, epruinose, hypothallus dark brown to black delimiting line along the margin, isidia and soredia absent. Apothecia sessile, immersed, 0.2-0.5(1.0) $\mathrm{mm}$ diameter, disc black, plane to convex, epruinose, oil globules absent, epithecium brown 24-36 $\mu \mathrm{m}$ thick, hymenium hyaline 78-92 $\mu \mathrm{m}$ high, hypothecium dark brown, 120-144 $\mu \mathrm{m}$ thick, exciple brown to dark brown 48$72 \mu \mathrm{m}$ thick, asci 8-spored, unitunicate, ascospores brown,4-celled, 3-transversely septate, 15-27 x 6-12 $\mu \mathrm{m}$ in size, wall uniformly thickened, surface ornamented, paraphyses hyaline, simple, globose at tips.

Chemistry: Thallus $\mathrm{K}^{+}$yellow turning red, $\mathrm{C}^{-}, \mathrm{KC}^{-}, \mathrm{PD}^{+}$ deep yellow.
Remark: B. lauricassiae resembles $B$. inornata in external morphology and chemistry but it is distinguished by possesses 4-celled, 3-transversely septate ascospores.

Distribution: India (Andhra Pradesh, Manipur and West Bengal).

Specimens examined: Manipur, Churachandpur, Singhat area, alt. ca. 4500 ft., 14.6.1979, K. P. Singh 550679, 550676 (CAL); West Bengal, 24-Paragnas, Saptagram, 3.10.1966, K. N. Roychowdhury 366 (CAL).

Buellia manipurensis Singh and Singh, Geophytology, 12 (1): 129, 1982.

Type: India; Manipur, Korong, Mao, on the way to Rubinimiah on rocks, alt. ca. 1960 m., June 5, 1979, K. P. Singh 550372 (CAL), (not seen).

Thallus saxicolous, crustaceous, $3-4 \mathrm{~cm}$ across, greyish brown, cracked to areolate, surface smooth, epruinose, hypothallus dark brown, thick delimiting line along the margin, isidia and soredia absent. Apothecia sessile, immersed, 0.3-1.5 mm diameter, disc dark brown, plane to convex, margin indistinct, epruinose, oil globules absent, epithecium brown 18-24 $\mu \mathrm{m}$ thick, hymenium hyaline 72-85 $\mu \mathrm{m}$ high, hypothecium dark brown, 60-96 $\mu \mathrm{m}$ thick, exciple dark brown along outer side and pale brown towards inner side $40-60 \mu \mathrm{m}$ thick, asci 8-spored, unitunicate, ascospores brown, 2-4-celled, straight to curved, constricted at septa, $18-27 \times 6-10 \mu \mathrm{m}$ in size, wall uniformly thickened, surface ornamented, paraphyses hyaline, simple, globose at tips.

Chemistry: Thallus $\mathrm{K}^{+}$yellow to reddish brown, $\mathrm{C}^{-}, \mathrm{KC}^{-}$, $\mathrm{PD}^{+}$deep yellow.

Remark: B. manipurensis resembles B. nubile in the nature of thallus reactions but the latter has smaller ascospores $(10-12 \times 4-5 \mu \mathrm{m})$ in size.

Distribution: India (Manipur).

Specimens examined: Manipur, Korang-Monjong hill, alt. ca. 3500-4000 ft., 1.11.1976, K. P. Singh 55016 (CAL); Churachandpur, Singhat area, alt. ca. 4500 ft., 14.6.1979, K. P. Singh 550681 (CAL).

Buellia meghalayensis S. Singh and D. D. Awasthi, Biol. Mem. 6 (2): 187.1981. 


\section{CHANDRA: REVISION OF THE LICHEN GENUS Buellia De Not. (FAMILY PHYSCIACEAE) FROM INDIA}

Type: India: Meghalaya, Mawsmai area, $7 \mathrm{Km}$. from Cherrapunji, alt. ca. 1000 m., Awasthi 7985 (AWAS), (not seen).

Thallus saxicolous, crustaceous, 2-3 cm across, yellowish grey to ashy grey, cracked to areolate, surface smooth, deeply fissured, epruinose, hypothallus black, delimiting line along the margin, isidia and soredia absent. Apothecia sessile, immersed, 0.5-1.0 $\mathrm{mm}$ diameter, disc black, plane to convex, slightly pruinose, margin indistinct, oil globules absent, epithecium brown 24-36 $\mu \mathrm{m}$ thick, hymenium hyaline 84-96 $\mu \mathrm{m}$ high, hypothecium dark brown 96-120 $\mu \mathrm{m}$ thick, exciple dark brown along outer side and pale brown towards inner side 60-72 $\mu \mathrm{m}$ thick, asci 8-spored, unitunicate, ascospores brown, 2-celled, ellipsoid, slightly curved, $18-27 \times 6-12 \mu \mathrm{m}$ in size, wall uniformly thickened, surface smooth, paraphyses hyaline, septate, branched, globose at tips.

Chemistry: Thallus $\mathrm{K}^{+}$yellow, $\mathrm{C}^{-}, \mathrm{KC}^{-}, \mathrm{PD}^{+}$deep yellow.

Remark: B.meghalayensis shows some resemblance to $B$. substigmea but the latter is distinguished by pinkish purple reaction by $\mathrm{HNO} 3$ in exciple, smooth ascospore surface and presence of atranorin and norstictic acid only.

Distribution: India (Manipur and Meghalaya).

Specimens examined: Manipur, Ukhrul, Shiroi hills, 10.6.1979 K. P. Singh 550573, (CAL).

Buellia morehensis Singh and Singh, Geophytology, 12 (1): 128. 1982.

Type: India, Manipur, Tegnopal, Moreh, on bark of tree, alt. ca. 1050 m., June 18, 1979 K. P. Singh 550855 (CAL), (not seen).

Thallus corticolous, crustaceous, 3-4 cm across, yellowish grey to grey brown, verruculose, verrucae prominent, epruinose, hypothallus indistinct but blackish at its junction with other thallus, isidia and soredia absent. Apothecia sessile to subsessile, immersed, 0.5-1.2 mm diameter, disc black, plane to convex, epruinose, margin indistinct, oil globules absent, epithecium brown $24 \mu \mathrm{m}$ thick, hymenium hyaline 84-96 $\mu \mathrm{m}$ high, hypothecium dark brown 108-120 $\mu \mathrm{m}$ thick, exciple dark brown along outer side and pale brown towards inner side 40-70 $\mu \mathrm{m}$ thick, asci 8-spored, unitunicate, ascospores brown, 2-celled, ellipsoid, 18-27 x 6-9 $\mu \mathrm{m}$ in size, wall uniformly thickened, surface ornamented, paraphyses hyaline, simple, globose at tips.
Chemistry: Thallus $\mathrm{K}^{+}$red, $\mathrm{C}^{-}, \mathrm{KC}^{-}, \mathrm{PD}^{+}$yellow.

Remark: B.morehensis resembles B. granularis Müll. Arg., B. inornata (Stirt.) Zahlbr. and B. stillingiana Stein. In external morphology. It differs from the first two taxa in ascospores morphology and from $B$. stillingianta in the colour of exciple and chemistry.

Distribution: India (Manipur).

Specimens examined: Manipur, Tegnopal, alt. ca. 50006000 ft., 21.6.1979, K. P. Singh 550954 (CAL); UkhrulHundung, alt. ca. 4700 ft., 5.11.1976, K. P. Singh 55200 (CAL).

\section{Buellia montana Magn., Bot. Notiser, 2: 199. 1954.}

Type: India, Uttarakhand, Naini Tal, China peak, alt. 7000 ft., on bark, 1949, P. N. Mehra (not traceable in UPS in litt. Where Magnusson's herbarium is now located), (not seen).

Thallus corticolous, crustaceous, 1-2 cm across, dusty to brownish grey, subverruculose, surface smooth, weakly fissured, epruinose, hypothallus absent, isidia and soredia absent. Apothecia sessile, immersed, 0.2-0.5mm diameter, disc black, plane to convex, epruinose, oil globules absent, epithecium brown 18-24 $\mu \mathrm{m}$ thick, hymenium hyaline 72-96 $\mu \mathrm{m}$ high, hypothecium dark brown 60-96 $\mu \mathrm{m}$ thick, exciple dark brown along outer side and pale brown towards inner side 48-60 $\mu \mathrm{m}$ thick, asci 8spored, unitunicate, ascospores brown, 2-celled, ellipsoid, 18-27 x 6-9 $\mu \mathrm{m}$ in size, wall uniformly thickened, surface ornamented, paraphyses hyaline, simple, globose at tips.

Chemistry: Thallus $\mathrm{K}^{-}, \mathrm{C}^{-}, \mathrm{KC}^{-}, \mathrm{PD}^{-}$.

Remark: B. montana resembles B. zahlbruckneri but the latter has a discontinuous, verruculose white thallus, which is $\mathrm{K}^{+}$yellowish. It also shows some resemblance with Buellia punctata, which can be distinguished from the former by \pm evanescent thallus and smaller $(9-16 \mu \mathrm{m})$ ascospores.

Distribution: India (Himachal Pradesh, Manipur and Uttarakhand).

Specimens examined: Manipur, Toribari, alt. ca. 35004000ft., 1.6.1979, K. P. Singh 550035(CAL).

Buellia palniensis S. Singh and D. D. Awasthi, Biol. Mem., 6 (2): 188.1981. 


\section{CHANDRA: REVISION OF THE LICHEN GENUS Buellia De Not. (FAMILY PHYSCIACEAE) FROM INDIA}

Type: India: Tamil Nadu, Palni hills, Hill view, below Perumalmalai, alt. ca. 1300 m., on rock, Awasthi \& Singh, 70.442 (LWU), (not seen).

Thallus corticolous, crustaceous, 2-3 cm across, dull, brownish grey, areolate, surface smooth, deeply fissured, epruinose, hypothallus indistinct, isidia and soredia absent. Apothecia sessile, immersed, 0. 5-1.0 mm diameter, disc black, plane to convex, epruinose, oil globules absent, epithecium brown 18-24 $\mu \mathrm{m}$ thick, hymenium hyaline $60-72 \mu \mathrm{m}$ high, hypothecium dark brown 96-108 $\mu \mathrm{m}$ thick, exciple dark brown along outer side and pale brown towards inner side 48-60 $\mu \mathrm{m}$ thick, asci 8spored, unitunicate, ascospores brown, 2-celled, ellipsoid, 18-24 x 6-12 $\mu \mathrm{m}$ in size, wall uniformly thickened, surface smooth, paraphyses hyaline, septate, branched, globose at tips.

Chemistry: Thallus $\mathrm{K}^{+}$yellow, $\mathrm{C}^{-}, \mathrm{KC}^{-}, \mathrm{PD}^{-}$.

Remark: $B$. palniensis shows some resemblance with $B$. sororioids and $B$. subsororioides but the latter two are distinguished by $\mathrm{K}^{+}$yellow to red and $\mathrm{PD}^{+}$yellow thalli.

Distribution: India (Manipur and Tamil Nadu).

Specimens examined: Manipur, Ukhrul, Shiroi hills, alt. 9000ft., 20.6.1979, K. P. Singh 550576 (CAL); Gungbi, alt. ca. 5500ft., 5.11.1976, K. P. Singh 55174 (CAL).

Buellia placodiomorpha Vain., Etud. Lich. Bresil, 1: 172. 1890. Imshaug, Farlowia, 4: 498, 1955.

Type: Brazil, adcorticem arbores prope Sepitiba in civ. Minarum, VAINIO 473 (TUR- VAIN), (not seen).

Thallus corticolous, crustaceous, 2-3 cm across, greyish brown to grey, subverruculose to evanescent, surface rough, weakly fissured, epruinose, hypothallus brownish black delimiting line along the margin, isidia and soredia absent. Apothecia sessile, immersed, 0. 2-0.5 mm diameter, disc black, plane to convex, epruinose, oil globules absent, epithecium brown $12 \mu \mathrm{m}$ thick, hymenium hyaline $72-84 \mu \mathrm{m}$ high, hypothecium dark brown 96-108 $\mu \mathrm{m}$ thick, exciple dark brown on outer margin and hyaline to brownish towards inner side 48-60 $\mu \mathrm{m}$ thick, asci 8spored, unitunicate, ascospores brown, 2-celled, polarilocular, placodiomorphic, 15-27 x 6-12 $\mu \mathrm{m}$ in size, wall uniformly thickened, surface ornamented, paraphyses hyaline, septate, branched, globose at tips.

Chemistry: Thallus $\mathrm{K}^{-}, \mathrm{C}^{-}, \mathrm{KC}^{-}, \mathrm{PD}^{-}$.
Remark: B. placodiomorphic is distinguished by 2-celled, polarilocular and placodiomorphic type of ascospores. It resembles $B$. placodiomorphoids but the latter is distinguished by $\mathrm{K}^{+}$yellow thalli.

Distribution: India (Manipur and Uttarakhand), Brazil and West Indies.

Specimens examined: Manipur, Tegnopal, alt. 5000ft., 21.6.1979, K. P. Singh 550921(CAL); Toribari, alt. ca. 3500-4000 ft., 1.6.1979, K. P. Singh 550047 (CAL).

Buellia posthabita (Nyl.)Zahlbr., Cat. Lich. Univ. 7: 390, 1931. Imshaug, Farlowia, 4: 499, 1955. Lecidea posthabita Nyl., Lich Nov. Zeland., 111, 1888.

Type: Nova Granata, Honda, 1861, Lindig, s. n. (H-NYL.), (not seen).

Thallus saxicolous, crustaceous, $2-3 \mathrm{~cm}$ across, brownish grey, subverruculose, surface rough, weakly fissured, epruinose, hypothallus indistinct, isidia and soredia absent. Apothecia sessile, immersed, 0.2-0.5 mm diameter, disc black, plane to convex, epruinose, oil globules absent, epithecium pale brown 24-36 $\mu \mathrm{m}$ thick, hymenium hyaline 96-108 $\mu \mathrm{m}$ high, hypothecium colourless to light brown 84-96 $\mu \mathrm{m}$ thick, exciple dark brown on outer side and pallid on inner side 60-84 $\mu \mathrm{m}$ thick, asci 8-spored, unitunicate, ascospores brown, 2celled, mischoblastiomorphic type, 24-27 x 12-15 $\mu \mathrm{m}$ in size, wall uniformly thickened, surface smooth, paraphyses hyaline, simple, branched, globose at tips.

Chemistry: Thallus $\mathrm{K}^{+}$yellowish, $\mathrm{C}^{-}, \mathrm{KC}^{-}, \mathrm{PD}^{-}$.

Remark: $B$. posthabita is distinguished by hypothallus indistinct and mischoblastiomorphic type of ascospores.

Distribution: India (Manipur), Nova Granata and West Indies.

Specimens examined: Manipur, Litan Sakkibung, alt. ca. 3500-4000 ft., 14.11.1979, K. P. Singh 54897 (CAL).

Buellia punctata (Hoffm.) Mass., Reicerch. Auton. Lich., 165, 1852. Sheard, Lichenologist, 2: 243, 1964. Verrucaria punctata Hoffm. Deutsch. Fl., 192, 1796.

Type: Massachusetts, Willey, (not seen).

Thallus corticolous, crustaceous, 3-4 cm across, whitish grey to glaucous white, subverruculose to granulose, surface smooth, weakly fissured, epruinose, hypothallus absent, isidia and soredia absent. Apothecia 


\section{CHANDRA: REVISION OF THE LICHEN GENUS Buellia De Not. (FAMILY PHYSCIACEAE) FROM INDIA}

sessile, immersed, 0.2-1.0 $\mathrm{mm}$ diameter, disc black, plane to convex, epruinose, oil globules absent, epithecium brown 18-24 $\mu \mathrm{m}$ thick, hymenium hyaline 60-72 $\mu \mathrm{m}$ high, hypothecium dark brown 96-108 $\mu \mathrm{m}$ thick, exciple dark brown long outer side and pale brown towards inner side 48-72 $\mu \mathrm{m}$ thick, asci 8-spored, unitunicate, ascospores brown, 2-celled, ellipsoid, 15-24 x 6-9 $\mu \mathrm{m}$ in size, wall uniformly thickened, surface ornamented, paraphyses hyaline, simple, globose at tips.

Chemistry: Thallus $\mathrm{K}^{+}$yellow, $\mathrm{C}^{-}, \mathrm{KC}^{-}, \mathrm{PD}^{-}$.

Remark: $B$. punctata resembles $B$. montana but the latter is distinguished by a smooth thallus and larger (18-27 $\mu \mathrm{m})$ ascospores. This is cosmopolitan in distribution.

Distribution: India (Himachal Pradesh, Jammu \& Kashmir, Manipur and West Bengal).

Specimens examined: Manipur, Tegnopal, alt. $5000 \mathrm{ft}$., 21.6.1979, K. P. Singh 550905 (CAL); West Bengal, 24Paraganas, Diamond harbour, 3.1.1972, K. N. Roychowdhury 2649 (CAL); Tongloo, alt. $10074 \mathrm{ft}$., 1.6.1966, K. N. Roychowdhury 54 (CAL).

\section{ACKNOWLEDGEMENT}

Author is thankful to the Director, Botanical Survey of India, Kolkata for providing necessary facilities and financial support and Dr. K. P. Singh, Ex. Additional Director, Botanical Survey of India, Central Circle, Allahabad for valuable guidance during the course of study.

\section{REFERENCES}

Awasthi D.D., 1988. A key to the Macrolichens of India and Nepal. J. Hattori. Bot. Lab., 65: 207-302.

Culberson C.F., 1972. Improved conditions and new data for the identification of lichen products by a standardized thin layer chromatographic method. J. Chromatography, 72: 113-125.

Singh K.P. and Singh S.R., 1982. Two new species of lichen genus Buellia from India. Geophytology, 12(1): 128-129.

Singh S.R. and Awasthi D.D., 1981. The lichen genus Buellia in India. Biol. Mem., 6(2): 169-196.

Walker F.G. and James P.W., 1980. A revised guide to michrochemical techniques for the identification of lichen products. Bull. British Lichen Soc. (Suppl.), 46: 13-29. 